\title{
Aplicación de la Terapia de Aceptación y Compromiso en pacientes con un Trastorno de la Conducta Alimentaria: un estudio piloto.
}

\section{Acceptance and commitment therapy for eating disorders: a pilot study with adolescent patients.}

Fecha de recepción: 05-07-2018

Fecha de aceptación: 12-07-2018

\author{
María Marco Cramer \\ Centro CREA \\ Yolanda Quiles Marcos \\ Departamento Psicología de la Salud. Universidad Miguel Hernández \\ Centro CREA \\ María José Quiles Sebastián \\ Departamento Psicología de la Salud. Universidad Miguel Hernández
}

\section{resumen/ahstract:}

La Terapia de Aceptación y Compromiso (ACT) se ha desarrollado como una alternativa conceptual conductista a los modelos cognitivo-conductuales y recientemente, se ha empezado a aplicar a los trastornos de la conducta alimentaria (TCA). El objetivo de este trabajo ha sido desarrollar, aplicar y evaluar un programa de intervención basado en la ACT en un grupo de pacientes con un TCA. Participaron 14 pacientes diagnosticadas de un TCA con una media de edad de 17.93 años $(D T=3.75)$. Se desarrolló y aplicó un programa de 12 sesiones basado en la ACT. Se evaluó la imagen corporal, calidad de vida, conciencia emocional, ansiedad y depresión, y aceptación, tanto antes como después de la intervención. Los análisis mostraron cambios en la apreciación corporal, calidad de vida así como en la atención plena. Estos resultados sugieren que disminuyeron las conductas evitativas, se redujo la insatisfacción corporal así como mejoró el conocimiento personal. En conclusión, los resultados apoyan la aplicación de la ACT en estas pacientes.

Acceptance and Commitment Therapy (ACT) has recently been developed as a conceptual and behavioral alternative to cognitive-behavioral models. The aim of this work has been to develop, apply and assess an intervention program based on CFT in a group of patients with eating disorders. The sample consisted of 14 patients from the Center for Emotional and Food Recovery, CREA. The mean age was 17.93 years (SD $=3.75)$. Different instruments were applied for the empirical evaluation of the treatment, measures of body image, quality of life, emotional awareness, anxiety and depression, and acceptance related to the psychological flexibility. The results showed changes in body appreciation, quality of life as well as in mindfulness. These results suggested that avoidant behaviors decreased, body dissatisfaction decreased

\section{palabras clave/keywords:}

Trastornos de la Conducta Alimentaria, Terapia de Aceptación y Compromiso, Atención Plena, Flexibilidad Psicológica. Eating Disorders, Acceptance and Commitment Therapy, mindfulness, psychological flexibility. 


\section{Introducción}

Los Trastornos de la Conducta Alimentaria (TCA) se caracterizan porque las personas que lo presentan tienen una conducta alterada de la ingesta alimentaria y/o comportamientos de control de peso (DSM 5, 2014). Son problemas con una larga duración y conllevan importantes consecuencias no solo a nivel físico sino también en el ámbito académico, familiar, emocional y social (NICE, 2017; Rojo-Moreno et al., 2017). Los TCA son trastornos complejos que implican un gran deterioro funcional, angustia emocional, riesgo de suicidio y alteración en el índice de masa corporal (IMC) (Stice, Marti, y Rohde, 2013).

Los TCA son comunes entre las mujeres jóvenes (Stice et al., 2013; Vaz-Leal et al., 2013). En las últimas décadas, ha habido un aumento en el riesgo de incidencia en el grupo poblacional comprendido entre las edades de 15 a 19 años (Rojo-Moreno et al., 2012; Smink, van Hoeke, y Hoek, 2012).

En cuanto al tratamiento psicológico eficaz para este problema de salud, las principales guías clínicas señalan las terapia cognitivo-conductual (TCC) y la terapia interpersonal (Guía de Práctica Clínica de los Trastornos de la Conducta alimentaria, 2009; NICE, 2017). Aunque se propone la TCC como la terapia de elección para tratar a los pacientes con un TCA, recientemente se ha desarrollado y se han aplicado otras terapias junto con la TCC, con el fin de optimizar los resultados, y complementar el tratamiento de los TCA abordando conceptos claves. Es así que, se están aplicando las terapias conocidas como Terapias de Tercera Generación, y entre ellas destacamos la Terapia de Aceptación y Compromiso (ACT) (Juarascio, Manasse, Schumacher, Espel, y Forman, 2016)

La ACT forma parte de la terapia cognitivo-conductual (TCC), pero al mismo tiempo contiene características muy distintivas, tratándose de un nuevo enfoque con un cambio de paradigma: más que centrarse en la eliminación de síntomas, se persigue cambiar la función de los eventos psicológicos y la relación de las personas con ellos a través de técnicas como la aceptación, la defusión y el mindfulness (Hayes, Strosahl, y Wilson, 2014). En este sentido, la ACT da un giro en el enfoque de la terapia: primero, no trata de cambiar o reducir pensamientos, sensaciones o recuerdos molestos, sino que se trata de alterar su función y generar flexibilidad en la regulación del comportamiento. La mayoría de las estrategias de ACT son cognitivas, su uso tiene como objetivo trabajar y empoderar los valores personales (Manlick, Cochran y Koon, 2012).

El aspecto fundamental en la ACT es el sufrimiento humano. En muchas ocasiones la felicidad es entendida como la ausencia de experiencias negativas (pensamientos y emociones). Desde la ACT el estado vivencial de la persona es comprendido como una serie de procesos psicológicos, donde se experimenta dolor y sufrimiento a través de las habilidades verbales de la persona. Las capacidades verbales de la persona facilitan las interacciones sociales forjando la autoestima. La conexión entre lenguaje, emociones y autoestima es una relación imprescindible para comprender la ACT. Esta conexión puede facilitar la evitación de situaciones dolorosos, indeseadas, pensamientos y emociones referidos como la evitación experiencial (Manlick et al., 2012; Masuda, Hill, Melcher, Morgan, y Twoghig, 2014). 
Tabla 1.- Procesos de la teoría de flexibilidad psicológica (Hayes, Luoma, Bond, Masuda y Lillis, 2006)

\begin{tabular}{|c|c|}
\hline $\begin{array}{l}\text { Proceso de flexibilidad } \\
\text { (e inflexibilidad) }\end{array}$ & Descripción \\
\hline $\begin{array}{l}\text { Aceptación } \\
\text { (Evitación experiencial) }\end{array}$ & $\begin{array}{l}\text { Acogimiento activo y consciente de los eventos privados } \\
\text { causados por la historia de uno mismo, sin intentos } \\
\text { innecesarios de cambiar su frecuencia o forma. }\end{array}$ \\
\hline $\begin{array}{l}\text { Defusión cognitiva } \\
\text { (Fusión cognitiva) }\end{array}$ & $\begin{array}{l}\text { Técnicas dirigidas a intentar cambiar la relación con los } \\
\text { pensamientos y a alterar las funciones indeseables de } \\
\text { pensamientos y otros eventos privados (sin cambiar } \\
\text { frecuencia o forma). El resultado de la defusión es la } \\
\text { reducción de credibilidad del pensamiento, o del apego/ } \\
\text { fusión a los eventos privados. }\end{array}$ \\
\hline $\begin{array}{l}\text { Yo como contexto } \\
\text { (Vinculación al yo conceptual) }\end{array}$ & $\begin{array}{l}\text { Self entendido como un contexto para el conocimiento } \\
\text { verbal, y no como el contenido del conocimiento. } \\
\text { Consciencia de las propias experiencias sin fusionarse } \\
\text { con ellas. }\end{array}$ \\
\hline $\begin{array}{l}\text { Atención al momento presente } \\
\text { (Atención rígida, bajo } \\
\text { autoconocimiento, pasado } \\
\text { conceptualizado y futuro } \\
\text { temido) }\end{array}$ & $\begin{array}{l}\text { Contacto libre de juicios, momento a momento, con } \\
\text { los eventos psicológicos y ambientales. El objetivo es } \\
\text { experimentar el mundo más directamente, de manera } \\
\text { que el comportamiento sea más flexible y, por tanto, las } \\
\text { acciones sean más consistentes con sus valores. }\end{array}$ \\
\hline $\begin{array}{l}\text { Valores } \\
\text { (Quiebra de valores, o } \\
\text { predominio de "valores" } \\
\text { de conformidad, fusión y } \\
\text { evitación) }\end{array}$ & $\begin{array}{l}\text { ACT ayuda a elegir direcciones vitales valiosas mientras } \\
\text { debilita los procesos verbales que pueden llevar a } \\
\text { elecciones basadas en la evitación, en el plegamiento } \\
\text { social o la fusión. Los procesos de aceptación, defusión, } \\
\text { atención al momento presente y yo como contexto } \\
\text { no son fines en sí mismos, sino que se emplean para } \\
\text { obtener una vida más consistente con los valores. }\end{array}$ \\
\hline $\begin{array}{l}\text { Compromiso con la acción } \\
\text { (Inactividad, impulsividad o } \\
\text { evitación persistentes) }\end{array}$ & $\begin{array}{l}\text { Desarrollo de patrones de acción relacionados con los } \\
\text { valores elegidos. Se pretende conseguir que se realicen } \\
\text { metas concretas en cuanto a cambio conductual, ya sean } \\
\text { éstas a corto, medio o largo plazo. El cambio conductual } \\
\text { dirige a entrar en contacto con las barreras psicológicas } \\
\text { abordadas en los otros procesos mencionados. }\end{array}$ \\
\hline
\end{tabular}


La ACT pretende mejorar la flexibilidad psicológica, entendida como la disposición activa a hacer contacto con la experiencia en el momento presente, de manera consciente, sin defensa y al servicio de aquello que es más importante para la persona (Orlando-Olaz, 2014). Por lo tanto, la reducción del síntoma, especialmente de experiencias internas tales como pensamientos angustiosos sobre el cuerpo, necesidad de atracones o sentirse deprimido se enfatiza en el tratamiento de la flexibilidad psicológica a través de seis procesos psicológicos (aceptación, momento presente, valores, conciencia, acción comprometida, yo contexto y defusión), representados en el Hexaflex y definidos en la tabla 1(Hayes, Luoma, Bond, Masuda, y Lillis, 2006). Cada uno de estos procesos puede ser entendido como un continuo, con el objetivo de pasar de un extremo patológico a la flexibilidad psicológica (Juarascio et al., 2013).

Desde la ACT los TCA se caracterizarían por una evitación experiencial, donde las pacientes centran su atención en su cuerpo y la comida como estrategia para evitar otros pensamientos y emociones que consideran fuera de su control (Berman, Boutelle, y Crow, 2009; Bluett et al., 2016; Butryn et al., 2012; Juarascio et al., 2016). Desde la ACT se trata de cambiar este patrón de atención en el cuerpo y la conducta alimentaria para dejar que emerjan otros comportamientos más adaptativos (Juarascio, 2011; Juarascio et al., 2013; Masuda et al., 2014). Estudios recientes en TCA muestran que entrenando y mejorando las estrategias de autocontrol emocional aumenta la oportunidad de la persona para regular sus emociones, y desde la calma y la posición no enjuiciadora la persona aprende a aceptar con conciencia plena sus experiencias negativas (Jennings y Apsche, 2014). Los estudios de revisión sistemática han sugerido que la ACT es eficaz como intervención en los TCA (Juarascio et al., 2013). Los resultados de estos estudios sugieren que los pacientes que experimentan mejoras en atención, aceptación y evitación emocional también presentan una reducción de los síntomas de TCA, además de mostrar cambios en los niveles de flexibilidad con respecto al inicio del tratamiento (Bluett et al., 2016; Butryn et al., 2013; Manlick et al., 2013).

Es por ello que, el objetivo de este estudio fue realizar un estudio piloto con el fin de desarrollar, aplicar y evaluar un programa de intervención basado en la ACT en personas diagnosticadas con un TCA.

\section{Material y métodos}

\section{Participantes}

Participaron 14 pacientes (13 chicas y 1 chico) con diagnóstico de TCA que acudían a tratamiento en el centro de día CREA, especializado en el abordaje de los TCA. La media de edad fue de 17.93 años $(D T=3.75)$. Respecto al diagnóstico, realizado por los profesionales médicos del centro siguiendo los criterios de DSM 5, un 57.1\% presentaba AN, un $14.3 \%$ BN, el $21.4 \%$ un Trastorno de Conducta Alimentaria No especificado (TCANE) y un $7.1 \%$ Trastorno por Atracón (TA). Respecto a los criterios de inclusión, además de tener diagnosticado un TCA, debía estar recibiendo tratamiento especializado en régimen de centro de día. Los criterios de exclusión fueron: presentar episodios de psicosis, ideación suicida o abuso de sustancias. 
En cuanto al nivel de estudios, un $28.6 \%$ cursaba E.S.O, un $28.6 \%$ estudios de bachiller, el $28.6 \%$ estudios universitarios, un $7.1 \%$ formación profesional y el $7.1 \%$ abandonaron los estudios de enseñanza secundaria obligatoria antes de finalizar. En relación con la duración del TCA, el rango osciló entre 11 y 94 meses $(M=32.64$ y $D T=24)$, encontrando que, el $42.9 \%$ presentaba el trastorno desde hacía menos de 24 meses. Un $57.1 \%$ de los participantes estaba en tratamiento farmacológico, siendo el más frecuente los antidepresivos, y un $64.3 \%$ presentaba comorbilidad con el TCA (depresión, trastorno de personalidad y trastorno de ansiedad).

\section{Instrumentos}

Imagen corporal:

- Escala de apreciación corporal (BAS) (Jáuregui y Bolaños, 2011). Este instrumento evalúa los aspectos positivos de la imagen corporal en adolescentes españoles. Consta de 13 ítems, con una escala de respuesta tipo Likert del 1 al 5, donde 1 es nunca y 5 es siempre, que valoran la existencia de una opinión favorable acerca de las características físicas, la aceptación de su cuerpo, respeto y atención a las necesidades de este a través de la adopción de comportamientos saludables, la autoprotección por rechazar los ideales de belleza presentados en los medios de comunicación y la forma o imperfecciones del cuerpo. Es un instrumento, de estructura unifactorial, con una adecuada consistencia interna $\alpha=.87$.

- Cuestionario de evitación corporal (BIAQ) (Jáuregui y Bolaños, 2011). Evalúa la flexibilidad cognitiva y la aceptación de la imagen corporal. Consta de 19 ítems que incluyen cinco alternativas de respuesta, de acuerdo con la frecuencia con que se realizan las conductas descritas en ellos. Es un instrumento multifactorial, con una consistencia interna de .9 , tanto para la escala total, como para las subescalas. El coeficiente Alpha en este trabajo fue de .75 .

- Satisfacción corporal. Para evaluar la satisfacción corporal se utilizó una única pregunta con una escala de respuesta Likert del 0 al 10, donde 0 es igual a "ninguna satisfacción" y 10 "mucha satisfacción”. La pregunta que se utilizó fue: “CCómo de satisfecha estás con tu cuerpo?”.

\section{Calidad de vida:}

- Cuestionario de salud SF-12 (Versión reducida del cuestionario SF-36) (Alonso, Prieto y Antó, 1995). Esta herramienta proporciona una medida subjetiva del estado de salud. Evalúa ocho aspectos de salud: funcionamiento físico, limitaciones en el rol por problemas físicos, funcionamiento social, dolor corporal, salud mental, limitaciones en el rol por problemas personales, vitalidad y salud general. Consta de un total de 12 ítems con respuesta tipo Likert del 1 al 5, donde 1 es excelente y 5 es mala. La consistencia interna en este estudio fue de $\alpha=.85$.

\section{Conciencia emocional:}

- Cuestionario de conciencia emocional (EAQ) (Samper, Mesurado, Richaud y Llorca, 2016). Es un autoinforme dirigido a niños y adolescentes. Consta de 30 ítems, agrupados en seis factores: diferenciar emociones, comunicar emociones 
verbalmente, no esconder emociones, conciencia corporal, atender emociones de otros y análisis de emociones propias. La respuesta es tipo Likert con tres opciones (desde $1=$ no es verdad, $2=$ a veces verdad, $3=$ verdad). Se obtuvo un índice de consistencia interna igual a .86 .

- Escala de conciencia y atención plena (MAAS). Se utilizó la versión española de Soler et al. (2012). Cuestionario unifactorial de 15 ítems diseñado para valorar una característica de la atención plena, la conciencia abierta. En este estudio el coeficiente alfa fue de .70 .

\section{Ansiedad y Depresión:}

- Escala de Ansiedad y Depresión Hospitalaria (HADS) (Terol, López-Roig, Rodríguez-Marín, Martín-Aragón y Pastor, 2007). Es un instrumento utilizado para evaluar síntomas de ansiedad y depresión en el ámbito hospitalario. Es una escala breve y adecuada en tanto que descarta los síntomas propios de la enfermedad. Consta de 14 ítems que se contestan en una escala de tipo Likert que va de 0 a 3 , donde 0 es todo el día y 3 es nunca. Presentó un índice de consistencia interna igual a .74 .

\section{Aceptación:}

- Cuestionario de aceptación y acción (AAQ) (Barraca, 2004). Consta de 16 ítems, en este trabajo ha sido utilizada la escala reducida, con 9 ítems. Estos ítems evalúan la evitación experiencial y la aceptación, dos aspectos claves dentro de la ACT. Estos están repartidos en 3 factores: factor I desbordamiento emocional, factor II inadecuada evaluación de los problemas y el factor III inadecuada respuesta a los problemas. La versión española de la AAQ se puede considerar una escala fiable y válida para la medida de la evitación experiencial y la aceptación psicológica en la población española. La consistencia interna de este instrumento fue igual a .42 .

\section{Procedimiento}

El programa se aplicó en el centro de día al cual asistían estas pacientes. Antes del inicio, se les explicó a las pacientes los objetivos y el procedimiento del estudio, se les invitó a participar de manera voluntaria, garantizando la confidencialidad de la información y el uso de los datos solo con fines de investigación.

El programa de intervención constaba de un total de 10 sesiones de una hora y cuarenta y cinco minutos de duración aproximada, con una periodicidad semanal, teniendo pues el programa una duración total de 2 meses y medio. Se empleó un formato grupal y el programa fue impartido por la misma terapeuta, formada y especializada en esta terapia.

Para el diseño de las sesiones se utilizaron distintos manuales de ACT (Hayes, 2013; Hayes y Smith, 2013). Se trató de seguir el protocolo de tratamiento de Juarascio et al., (2013), interviniendo sobre los aspectos centrales de esta teoría; los seis procesos pueden resumirse en tres objetivos principales: estar dispuesto (aceptación y defusión), centrado (momento presente y yo contexto) y comprometido (valores y acción comprometida). En la tabla 2 se exponen los objetivos y contenidos de cada una de las sesiones. 
Tabla 2.- Objetivos y contenidos de las sesione

\begin{tabular}{|c|c|c|}
\hline Sesión & Objetivos & Materiales \\
\hline $\begin{array}{l}\text { 0. Evaluación } \\
\text { inicial. }\end{array}$ & $\begin{array}{l}\text { Informar sobre el tratamiento. } \\
\text { Evaluar y recoger los datos sobre el } \\
\text { estado anímico de los pacientes antes de } \\
\text { la intervención. }\end{array}$ & $\begin{array}{l}\text { Batería de cuestionarios. } \\
\text { Consentimiento informado. }\end{array}$ \\
\hline $\begin{array}{l}\text { 1. Presentación } \\
\text { ACT y Modelo } \\
\text { Matrix. }\end{array}$ & $\begin{array}{l}\text { Explicar la Terapia de Aceptación y } \\
\text { Compromiso, como sus } 6 \text { procesos } \\
\text { básicos. } \\
\text { Explicar el Modelo Matrix. }\end{array}$ & \\
\hline $\begin{array}{l}\text { 2. Proceso } \\
\text { Cognitivo. }\end{array}$ & Identificar pensamientos negativos. & $\begin{array}{l}\text { Ejercicio "La carga del tren de la } \\
\text { mente" (Hayes, S., y Smith, S., } \\
\text { 2013). }\end{array}$ \\
\hline $\begin{array}{l}\text { 3. Proceso } \\
\text { Cognitivo I. }\end{array}$ & $\begin{array}{l}\text { Identificar pensamientos negativos. } \\
\text { Entrenar las técnicas de defusión } \\
\text { cognitiva. }\end{array}$ & $\begin{array}{l}\text { Ejercicio "Hojas flotando en la } \\
\text { corriente" (Hayes, S., y Smith, S., } \\
2013 \text { ). } \\
\text { Manual de técnicas de defusión } \\
\text { cognitiva. }\end{array}$ \\
\hline $\begin{array}{l}\text { 4. Aceptación: } \\
\text { Aceptar y } \\
\text { comprender } \\
\text { eventos } \\
\text { inesperados. }\end{array}$ & $\begin{array}{l}\text { Conocer el concepto Aceptación y } \\
\text { Evitación experiencial. } \\
\text { Conocer autoconceptualizaciones. }\end{array}$ & $\begin{array}{l}\text { Plantilla sesión } 4 . \\
\text { Ejercicio "Reinterpretar la propia } \\
\text { historia" (Hayes, S., y Smith, S., } \\
\text { 2013). }\end{array}$ \\
\hline $\begin{array}{l}\text { 5. Metáfora de } \\
\text { ajedrez. }\end{array}$ & $\begin{array}{l}\text { Distinguir los contenidos del yo y el yo- } \\
\text { contexto. } \\
\text { Promover la práctica que permite situarse } \\
\text { en la posición del tablero. }\end{array}$ & $\begin{array}{l}\text { Ejercicio "Metáfora de ajedrez" } \\
\text { (Hayes, S., Stroshal, K., y Wilson, } \\
\text { K., 2014). }\end{array}$ \\
\hline $\begin{array}{l}\text { 6. Contacto con el } \\
\text { momento } \\
\text { presente. }\end{array}$ & Tomar contacto con el aqui y ahora. & $\begin{array}{l}\text { Ejercicio "Estar donde estás" } \\
\text { (Hayes, S., y Smith, S., 2013). }\end{array}$ \\
\hline $\begin{array}{l}\text { 7. Estar } \\
\text { dispuesto. }\end{array}$ & Promover el "estar dispuesto". & $\begin{array}{l}\text { Ejercicio "Dolor" (Hayes, S., y Smith, } \\
\text { S., 2013). } \\
\text { Ejercicio "Qué evito" (Hayes, S., y } \\
\text { Smith, S., 2013). }\end{array}$ \\
\hline $\begin{array}{l}\text { 8. Valores y } \\
\text { acciones } \\
\text { valoradas. }\end{array}$ & $\begin{array}{l}\text { Conocer valores en los principales } \\
\text { ámbitos de la vida. }\end{array}$ & $\begin{array}{l}\text { Video "Pensamientos pasajeros" } \\
\text { (Hayes, S., Stroshal, K., y Wilson, } \\
\text { K., 2014). } \\
\text { Ejercicio "La diana." (Hayes, S., y } \\
\text { Smith, S., 2013). }\end{array}$ \\
\hline $\begin{array}{l}9 . \text { Valores y } \\
\text { acciones } \\
\text { valoradas II. }\end{array}$ & $\begin{array}{l}\text { Conocer valores en los principales } \\
\text { ámbitos de la vida. } \\
\text { Establecer metas. }\end{array}$ & $\begin{array}{l}\text { Ejercicio "Hoja de valores, barreras } \\
\text { y estrategias" (Hayes, S., y Smith, } \\
\text { S., 2013). }\end{array}$ \\
\hline $\begin{array}{l}\text { 10. Mindfulness y } \\
\text { contrato de } \\
\text { acción. }\end{array}$ & $\begin{array}{l}\text { Entrenar habilidades de atención plena } \\
\text { diaria. } \\
\text { Comprometerse a la acción. }\end{array}$ & $\begin{array}{l}\text { Ejercicio "El globo que se infla" } \\
\text { (Hayes, S., Stroshal, K., y Wilson, } \\
\text { K., 2014). } \\
\text { Contrato de acción y aceptación. }\end{array}$ \\
\hline $\begin{array}{l}\text { 11. Evaluación } \\
\text { final. }\end{array}$ & $\begin{array}{l}\text { Finalización del grupo de tratamiento. } \\
\text { Recoger los datos del estado anímico de } \\
\text { los pacientes tras la intervención. }\end{array}$ & Batería de cuestionarios. \\
\hline
\end{tabular}


Cabe señalar que la aplicación del programa se realizó de manera complementaria y simultánea a las intervenciones psicológicas y psiquiátricas que recibían las pacientes en el centro de día. El tratamiento que estas pacientes recibían en el centro de día incluía, además de la normalización de la alimentación en el comedor terapéutico, la asistencia y participación en otros talleres grupales dirigidos a mejorar su autoestima, imagen corporal, gestión de emociones, conciencia de enfermedad y motivación al cambio, sus conocimientos de nutrición y habilidades sociales. El programa se aplicó en el centro de día CREA.

\section{Tipo de diseño}

Para este estudio se ha empleado un diseño cuasi-experimental de grupo pretest-postest $(D G P P)$.

\section{Análisis de datos}

El tratamiento estadístico de los datos se realizó mediante el paquete estadístico SPSS v.22. Primero, se procedió a la comprobación de los supuestos de aplicación: normalidad, mediante la prueba estadística Kolmogorov-Smirnov, e independencia, con la prueba estadística de Rachas. Obteniendo resultados favorables para realizar una $t$-Student, se calcularon la diferencia entre medias como prueba paramétrica en muestras relacionadas, así como la potencia de contraste de las diferentes pruebas. Finalmente, se calculó el tamaño del efecto $d$ Cohen, considerando la clasificación de los valores $d=.20$ pequeño, $d=.50$ medio y $d=.80$ grande.

\section{Resultados}

Los resultados obtenidos se presentan en la tabla 3. Respecto a la variable Imagen Corporal, tras la intervención, los análisis mostraron un aumento de la puntuación media en la escala BAS, lo que refleja una opinión más favorable respecto a las características físicas del propio cuerpo. En el caso del BIAQ, la puntuación media de la escala total disminuyó de forma significativa cómo también se produjo una disminución significativa en las subescalas Ropa, Actividad Social, Restricción y en Acicalarse. Estos resultados reflejan un descenso en la evitación corporal y, por tanto, un aumento en la aceptación de la propia imagen.

En cuanto a Calidad de Vida, los resultados indicaron que mejoró positivamente la percepción de su salud mental, así como el papel de la salud física en las actividades del día a día.

Respecto a la variable Conciencia Emocional, los análisis mostraron un aumento significativo de la puntuación media tras la intervención, también se encontraron diferencias significativas en dos de sus subescalas: diferenciar emociones y no esconder emociones, encontrando que mejoró la identificación emocional y la expresión de esta. En el caso de la escala MAAS, que evalúa conciencia y atención plena, los análisis mostraron diferencias significativas tras la intervención, lo que indicaría una mejoría en la atención del momento presente.

Respecto a las dimensiones de ansiedad y depresión, los resultados mostraron diferencias significativas en la puntuación media de la subescala ansiedad, resultando ser ésta mayor al finalizar la intervención. 
No se encontraron diferencias significativas en ninguna de las subescalas del instrumento que evalúa aceptación y acción (AAQ).

Tabla 3.- Resultados (I)

\begin{tabular}{|c|c|c|c|c|c|}
\hline Variable & & $\begin{array}{c}\text { Pre } \\
M(D T)\end{array}$ & $\begin{array}{c}\text { Post } \\
M(D T)\end{array}$ & $t$ & $\begin{array}{c}\text { Tamaño } \\
\text { del efec- } \\
\text { to } d\end{array}$ \\
\hline IMC & & $21.50(4.46)$ & $21.40(4.38)$ & .80 & .02 \\
\hline \multicolumn{6}{|l|}{ Satisfacción } \\
\hline Corporal & & $3.64(1.91)$ & $5.14(2.54)$ & -4.17 & .66 \\
\hline$B A S$ & & $29.29(8.07)$ & 38.57 (11.78) & $-5.32^{\star *}$ & .92 \\
\hline \multirow[t]{5}{*}{$B I A Q$ (total) } & & $46.43(17.73)$ & $52.79(14.54)$ & $4.37^{* \star}$ & .84 \\
\hline & Ropa & $18.57(7.15)$ & $13(6.65)$ & $3.62^{\star *}$ & .81 \\
\hline & Actividad Social & $14.14(8.44)$ & $9.86(7.67)$ & $2.86^{\star}$ & .53 \\
\hline & $\begin{array}{l}\text { Restricción } \\
\text { Alimentaria }\end{array}$ & $4.14(4.69)$ & $2.07(2.67)$ & $3.07^{* *}$ & .54 \\
\hline & Acicalarse & $9.57(2.21)$ & $7.86(2.77)$ & $2.57^{*}$ & .68 \\
\hline \multirow[t]{9}{*}{ SF-12 (total) } & & $30.00(1.96)$ & $30.64(2.27)$ & -1.09 & .30 \\
\hline & Salud General & $3.29(1.07)$ & $4.14(1.03)$ & -1.83 & .80 \\
\hline & $\begin{array}{l}\text { Funcionamiento } \\
\text { Físico }\end{array}$ & $5.07(1.07)$ & $5(.08)$ & .18 & .09 \\
\hline & Rol Físico & $2.79(.89)$ & $3.36(.93)$ & $-2.83^{* *}$ & .63 \\
\hline & Vitalidad & $3.43(1.40)$ & $3.36(.84)$ & .21 & .06 \\
\hline & $\begin{array}{l}\text { Funcionamiento } \\
\text { Social }\end{array}$ & $3.07(1.32)$ & $3.64(1.08)$ & -1.52 & .47 \\
\hline & Rol Emocional & $2.5(.76)$ & $3.07(1.33)$ & -1.96 & .52 \\
\hline & Salud Mental & $7.29(.91)$ & $5.79(2.22)$ & $2.18^{*}$ & .88 \\
\hline & Dolor Corporal & $2.57(1.74)$ & $1.5(.52)$ & 2.02 & .83 \\
\hline
\end{tabular}


Tabla 3.- Resultados (II)

\begin{tabular}{|c|c|c|c|c|c|}
\hline Variable & & $\begin{array}{c}\text { Pre } \\
M(D T)\end{array}$ & $\begin{array}{c}\text { Post } \\
M(D T)\end{array}$ & $t$ & $\begin{array}{c}\text { Tamaño } \\
\text { del efec- } \\
\text { to } d\end{array}$ \\
\hline \multirow{7}{*}{$\begin{array}{l}E A Q \text { (to- } \\
\text { tal) }\end{array}$} & & $67.07(4.97)$ & $63.00(5.43)$ & $2.54^{*}$ & .78 \\
\hline & Diferenciar Emociones & $15.21(3.76)$ & $12.42(3.97)$ & $3.29^{* *}$ & .72 \\
\hline & $\begin{array}{l}\text { Comunicar Emo Ver- } \\
\text { bal }^{1}\end{array}$ & $6.07(.92)$ & $5.85(.86)$ & .90 & .25 \\
\hline & No esconder & $11.28(2.94)$ & $10.07(2.58)$ & $2.30^{*}$ & .44 \\
\hline & Conciencia Corporal & $12.28(1.14)$ & $11.71(1.48)$ & 1.12 & .44 \\
\hline & Emociones Otros & $10.21(.80)$ & $10.42(.64)$ & -.71 & .29 \\
\hline & Análisis Emoc Propias & $12(2.35)$ & $12.5(2.10)$ & -1.24 & .22 \\
\hline MAAS & & $46.00(11.88)$ & $51.86(14.85)$ & $-1.95^{*}$ & .43 \\
\hline \multirow{3}{*}{$\begin{array}{l}\text { HADS } \\
\text { (total) }\end{array}$} & & $19.64(3.63)$ & $18.86(2.85)$ & .94 & .24 \\
\hline & Escala de Ansiedad & $10.71(3.52)$ & $9.14(2.54)$ & $2.96^{* *}$ & .51 \\
\hline & Escala de Depresión & $8.93(2.99)$ & $9.71(3.12)$ & -1.30 & .25 \\
\hline \multirow{5}{*}{$\begin{array}{l}A A Q \text { (to- } \\
\text { tal) }\end{array}$} & & $40.21(4.99)$ & $38.79(6.05)$ & .96 & .25 \\
\hline & Desbordamiento Emoc & $24.42(4.77)$ & $21.78(5.65)$ & 1.72 & .50 \\
\hline & Inadecuada Eval Probl ${ }^{2}$ & $8.85(2.41)$ & $8.92(2.36)$ & -.11 & .02 \\
\hline & Inadecuada Resp & $6.92(1.73)$ & $8.07(2.81)$ & -1.79 & .50 \\
\hline & Probl $^{3}$ & & & & \\
\hline
\end{tabular}

${ }^{* *} p<.01,{ }^{*} p<.05$

1 Comunicar Emociones Verbalmente: Variable del cuestionario EAQ

2 Inadecuada Evaluación de los Problemas: Variable del cuestionario $A A Q$

3 Inadecuada Respuesta a los Problemas: Variable del cuestionario $A A Q$ 


\section{Discusión}

El objetivo de este trabajo fue desarrollar y aplicar un programa de intervención basado en la ACT en un grupo de pacientes con un diagnóstico de TCA, y analizar su eficacia. Tras analizar los resultados se observa que la aplicación del programa produce una mejoría clínica en los pacientes que recibieron la intervención.

Respecto a la imagen corporal, se observó un cambio en la actitud frente al aspecto físico tras la intervención, en su apreciación corporal. Se produjo una reducción de la insatisfacción y de la distorsión corporal, consiguiendo disminuir pensamientos erróneos sobre ésta y sustituirlos por otros más adaptativos y objetivos. En cuanto a la evitación corporal, los resultados mostraron una disminución de las conductas evitativas relacionados con la ropa y la restricción alimentaria. Cabe resaltar que desde el paradigma de la ACT este resultado es positivo, ya que no se busca eliminar la evitación experiencial sino experimentar y valorar las situaciones generadoras de esta conducta y confrontarlas (Hayes et al., 2014). Durante el desarrollo del programa siguiendo los fundamentos de la ACT, los pacientes fueron alentados y motivados verbalmente para participar en diversas actividades que tendían a evitar, lo que podría estar relacionado con los resultados obtenidos.

En la calidad de vida, los participantes presentaron mejores resultados en el Rol Físico y Salud Mental tras la participación en los talleres. Estos resultados se pueden interpretar como un menor impedimento a la hora de realizar actividades de la vida diaria y un menor sentimiento de angustia y depresión durante todo el tiempo.

Uno de los objetivos de la ACT es mejorar la conciencia emocional con la finalidad de mejorar la aceptación y el conocimiento personal. Al igual que en otros estudios recientes (Manlick et al., 2013), tras la aplicación de estrategias basadas en la aceptación del problema, se produjeron mejoras significativas en la conciencia plena. En concreto, los participantes mejoraron en la diferenciación y comunicación de emociones, así como en no ocultarlas.

Los análisis también mostraron puntuaciones superiores en la atención plena o mindfulness tras finalizar la intervención. Estos resultados sugieren que aumentó la frecuencia de situaciones en las que los participantes tenían la conciencia abierta o receptiva de la atención y la atención centrada en lo que estaba ocurriendo.

No obstante, no se obtuvieron diferencias significativas en evitación experiencial y en aceptación, evaluadas con el instrumento $A A Q$. Este resultado podría deberse a que, a pesar de que este instrumento ha demostrado su efectividad con una muestra heterogénea está limitado por las características de la muestra general, y la redacción en negativo de sus ítems, siendo sensibles a la confusión por parte del lector; lo que podría explicar porque no ha dado resultados significativos en esta población clínica.

Los resultados de este estudio piloto sugieren que, en general, la ACT puede resultar efectiva, para el tratamiento de algunos aspectos de los TCA. La TCC se centra en reducir los síntomas del TCA, lo cual es verdaderamente difícil debido a la naturaleza egosintónica que presentan las cogniciones propias de estos trastornos (Juarascio et al., 2013). Por tanto, 
la ACT puede resultar eficaz porque su objetivo no es cambiar esas cogniciones, sino ser capaces de experimentarlas sin responder a ellas restringiendo comida, sin estar de acuerdo con ellas, sin discutir con ellas (Mac Neil y Montemarano, 2015). Los participantes informaron que estaban satisfechos y que el contenido les resultó útil para reconocer los valores personales y aprender estrategias para el cambio comportamental. La satisfacción podemos asociarla al hecho de ser un programa basado no solo en la sintomatología alimentaria sino también a los aspectos más relevantes de su día a día (Mac Neil y Hudson, 2018).

En lo que respecta a las limitaciones halladas en el presente trabajo, es necesario considerar, en primer lugar, que los resultados deben ser interpretados de forma cautelosa, ya que se trata de un estudio piloto, y la intervención se ha desarrollado de forma integrada en el enfoque multidisciplinar de intervenciones del centro de día. Como se ha señalado anteriormente, en el centro de día se realizaban grupos de autoestima e imagen corporal, cuya participación podría haber influido en los resultados encontrados en este estudio. No obstante, en el centro no se realizaban sesiones relacionadas con otros componentes de esta terapia como la flexibilidad psicológica y la conciencia plena. Asimismo, la muestra clínica a la que se ha podido acceder presenta un tamaño pequeño y no se ha contado con un grupo de control, por lo que sería recomendable seguir utilizando esta terapia en grupos de mayor tamaño y utilizar un grupo control con el que comparar los resultados. De igual modo, se considera de gran interés la realización de un seguimiento a los seis meses post-intervención, llevado a cabo por investigadores no implicados en la aplicación de la intervención, con el fin de analizar si las mejoras obtenidas por el programa se mantienen con el tiempo.

Un tratamiento más prolongado, un mayor enfoque en los factores de mantenimiento del problema podrían mejorar los resultados de ACT (Parling, et al., 2016).

A pesar de estas limitaciones, los resultados de este trabajo son prometedores respecto a la eficacia de ACT en la intervención en el ámbito de los TCA. Este tipo de estrategias clínicas pueden ser útiles en trastornos comúnmente resistentes como los TCA, porque no se centran exclusivamente en las conductas patológicas pero si en la dirección de los problemas que se presentan en su vida diaria; siendo expresados en la relación terapéutica (Martín-Murcia, Cangas y Martín Del Águila, 2016). La ACT se presenta como una alternativa, que acumula evidencia a su favor, para la intervención y tratamiento de estos trastornos tan complejos.

\section{REFERENGIAS}

Alonso, J., Prieto, L., y Antó, J.M. (1995). La versión española del SF-36 Health Survey (Cuestionario de salud SF-36): un instrumento para la medida de los resultados clínicos. Medicina Clínica, 104(20), 771-776.

Barraca, J. (2004). Spanish Adaptation of the Acceptance and Action Questionnaire (AA0). International Journal of Psychology and Psychological Therapy, 4, 505-515.

Berman, M. I., Boutelle, K., y Crow, S. (2009). A case series investigating acceptance and commitment therapy as a treatment for previously treated, unremitted patients with anorexia nervosa. European Eating Disorders Review, 17(6), 426-434. 
Bluett, E. J., Lee, E. B., Simone, M., Twohig, M. P., Lesengrav, T. y Quakenbush, B. (2016). The role of body image psychological flexibility on the treatment of eating disorders in a residential facility. Eating Disorder, 23, 150-155.

Butryn, M. L., Juarascio, A., Shaw, J., Kerrigan, S.C., Clark, V., O'Planick, A. y Forman, E. M. (2013). Mindfulness and its relationship with eating disorders symptomatology in women receiving residential treatment. Eating Behaviors, 14, 13-16.

Grupo de trabajo de la Guía de práctica clínica sobre trastornos de la conducta alimentaria. Guía de práctica clínica sobre trastornos de la conducta alimentaria. Ministerio de Sanidad y Consumo. Agència d'Avaluació de Tecnologia i Recerca Mèdiques de Cataluña; 2009. http://www.guiasalud.es/GPC/GPC_440_Tt_Conduc_Alim_compl_(4_jun).pdf

Hayes, S. (2013). The instructor's manual: acceptance and commitment therapy: values and action with steven hayes, PhD. Mill Valley: Psychotherapy.net.

Hayes, S. C., Luoma, J. B., Bond, F. W., Masuda, A., y Lillis, J. (2006). Acceptance and Commitment Therapy: Model, Processes and Outcomes. Behavioral Research Therapy, 44, 1-25.

Hayes, S., Strosahl, K., y Wilson, K. (2014). Terapia de aceptación y compromiso. Proceso y práctica del cambio consciente (Mindfulness). Bilbao: Desclée de Brouwer.

Hayes, S., y Smith, S. (2013). Sal de tu mente, entra en tu vida. Bilbao: Desclée de Brouwer.

Jáuregui, I., y Bolaños, P. (2011). Body image and quality of life in a Spanish population. International Journal of General Medicine, 4, 63-72.

Jennings, J. L., y Apsche, J. A. (2014). The evolution of a fundamentally mindfulness-based treatment methodology: from DBT and ACT to MDT and beyond. International Journal of Behavioral Consultation and Therapy, 9(2), 1-3.

Juarascio, A. (2011). Acceptance and commitment therapy as a novel treatment for eating disorders: an initial test of efficacy and mediation. Pensilvania: Drexel University

Juarascio, A., Manasse, S., Schumacher, L., Espel, H. y Forman, E. (2016). Developing an Acceptance-Based Behavioral Treatment for Binge Eating Disorder: Rationale and Challenges. Cognitve and Behavioral Practice, 24, $1-13$.

Juarascio, A., Shaw, J., Forman, E.M., Timko, C.A., Herbert, J.D., Butryn, M.L., y Lowe, M. (2013). Acceptance and commitment therapy for eating disorders: clinical applications of a group treatment. Journal of Contextual Behavioral Science, 2, 85-94.

Mac Neil, B. y Hudson, C. (2018). Patient experience and satisfaction with Acceptance and Commitment therapy delivered in a complimentary open group format for adults with eating disorders. Journal of Patient Experience, 5(3), 189-194.

Mac Neil, B. y Montemarano, V. (2015). Acceptance and commitment therapy for adult eating disorders: Preliminary outcomes and patient satisfaction for a values based approach to treatment and recovery. Conference: Annual Canadian Psychological Association Conference.

Manlick, C. F., Cochran, S. V., y Koon, J. (2013). Acceptance and commitment therapy for eating disorders: rationale and literature review. Journal of Contemporary Psychotherapy, 43, 115-122.

Martín-Murcia, F., Cangas Díaz, A y Martín Del Águila, C. (2016). Integrating third generation psychological therapies in three cases of personality and eating disorders. 23rd International Symposium about Current Issues and Controversies in Psychiatry, Barcelona.

Masuda, A., Hill, M. L., Melcher, H., Morgan, J., y Twohig, M. P. (2014). Acceptance and commitment therapy for women diagnosed with binge eating disorder: A case-series study. Psychology Faculty Publications, 89, 1-35.

National Institute for Clinical Excellence (NICE). (2017). Eating disorders core interventions in the tratment and management of anorexia nervosa, bulimia nervosa and related eating disorders (Clinical Guideline No. 9). London: The British Psychological Society. The Royal College of Psychiatricts.

Orlando-Olaz, F. (2014). Aplicaciones de la terapia de aceptación y compromiso en el abordaje de la bulimia. Universidad Nacional de Córdoba: Facultad de Psicología. 
Parling, T., Cernvall, M., Ramklint, M., Holmgren, S. y Ghaderi, A. (2016). A randomised trial of Acceptance and Commitment Therapy for Anorexia Nervosa after daycare treatment, including five-year follow-up. BMC Psychiatric, 16(1).

Rojo-Moreno, L., Iranzo, C., Gimeno, N., Barberá, M. A., Rojo, L. M. y Liviana, L. (2017). Influencias genéticas y ambientales en rasgos psicológicos y actitudes alimentarias en una población escolar española. Revista de Psiquiatría y Salud Mental, 10, 134-142.

Rojo-Moreno, L., Plumed, J., Conesa, L., Vaz, F., Diaz, M., Rojo, L. y Livianas, L. (2012). Eating disorders: Considerations on nosology, etiology and treatment in the XXI century. Revista de Psiquiatría y Salud Mental, 5(3), $197-204$.

Samper, P., Mesurado, B., Richaud, M. C. y Llorca, A. (2016). Validación del Cuestionario de Conciencia Emocional en Adolescentes Españoles. Interdisciplinaria, 13, 1-14.

Soler, J., Tejedor, R., Feliu-Soler, A., Pascual, J. C., Cebolla, A. y Soriano, J. (2012). Propiedades psicométricas de la versión española de la escala Mindful Attention Awareness Scale (MAAS). Actas Españolas de Psiquiatría, $40,19-26$.

Stice, E., Marti, C., y Nathan, R. (2013). Prevalence, incidence, impairment, and course of the proposed DSM-5 eating disorder diagnoses in an 8-year prospective community study of young women. Journal of Abnormal Psychology, 122(2), 445-457.

Terol, M. C., López-Roig, S., Rodríguez-Marín, J., Martín-Aragón, M. y Pastor, M. A. (2007). Ansiedad y Estrés, 13, 163-176.

Vaz-Leal, F J., Rodríguez-Santos, L., García-Herráiz, M A., Chimpen, C. A., Rojo, L., Beato, L., y Ramos, M. J. (2013). The role of depression and impulsivity in the psychopathology of bulimia nervosa. Revista de Psiquiatría $y$ Salud Mental, 7(1), 25-31. 